\title{
Elation Laguerre planes of order 16 are ovoidal
}

\author{
Günter F. Steinke
}

\begin{abstract}
We conduct a computer search for elation Laguerre planes of order 16 and show that each such plane is ovoidal. The search results also confirm the numbers of hyperovals through certain points in the dual translation planes of order 16 given in $[15]$.
\end{abstract}

MSC 2000: 51E25, 51B15.

\section{Introduction and result}

A finite Laguerre plane $\mathcal{L}$ of order $n$ is an orthogonal array of strength 3 on $n$ symbols (levels), $n+1$ constraints and index 1, cf. [1], or equivalently, a transversal design $\mathrm{TD}_{1}(3, n+1, n)$. Since we have a more geometric point of view we rather use the term Laguerre plane instead of orthogonal array or transversal design, see section 2 for an explicit definition.

Models of finite Laguerre planes can be obtained as follows. Let $\mathcal{O}$ be an oval in the Desarguesian projective plane $\mathcal{P}_{2}=\mathrm{PG}(2, q), q$ a prime power. Embed $\mathcal{P}_{2}$ into 3 dimensional projective space $\mathcal{P}_{3}=\mathrm{PG}(3, q)$ and let $v$ be a point of $\mathcal{P}_{3}$ not belonging to $\mathcal{P}_{2}$. Then $P$ consists of all points of the cone with base $\mathcal{O}$ and vertex $v$ except the point $v$. Generators are the traces of lines of $\mathcal{P}_{3}$ through $v$ that are contained in the cone. The blocks of the design - usually called circles - are obtained by intersecting $P$ with planes of $\mathcal{P}_{3}$ not passing through $v$. In this way one obtains an ovoidal Laguerre plane of order $q$. If the oval $\mathcal{O}$ one starts off with is a conic, one obtains the Miquelian Laguerre plane of order $q$. All known finite Laguerre planes of odd order are Miquelian and all known finite Laguerre planes of even order are ovoidal. In fact, it is a long standing problem whether or not these are the only finite Laguerre planes. (There are many non-ovoidal infinite Laguerre planes though.)

Some partial results in this direction were obtained by combining the classifications for finite projective planes of small orders and their ovals, see section 2 for a description of the relation of Laguerre planes to projective planes and ovals. In this way it was shown that a Laguerre plane of order at most nine must be ovoidal, compare [3], [19] and [20]. The next interesting case is order 16. In [7] is was shown that translation Laguerre planes of order 16 must be ovoidal. (Such a plane is a Laguerre plane $\mathcal{L}$ with a distinguished generator $G$ such that each derived affine plane at a point of $G$ is a translation plane, and 
furthermore each translation in these derived planes is induced by an automorphism of $\mathcal{L}$ that fixes $G$ pointwise.)

Finite elation Laguerre planes were introduced in [21] and [18], see the following section for a description of the structure of finite elation Laguerre planes. They are characterized by the existence of a group of automorphisms that acts trivially on the set of generators and regularly on the set of circles. This group of automorphisms, which we call the elation group of the Laguerre plane, is unique and potentially plays a role analogous to the translation group of finite translation planes. In fact, elation Laguerre planes are linked to dual translation planes since such Laguerre planes can be described as dual translation planes with collections of certain ovals, see section 2 .

Every ovoidal Laguerre plane is an elation Laguerre plane, but there are infinite nonovoidal elation Laguerre planes; see, for example, [17]. Hence elation Laguerre planes form a proper generalization of the notion of ovoidal Laguerre planes. In [11], elation Laguerre planes were further characterized as weakly Miquelian Laguerre planes, that is, those Laguerre planes in which a certain variation M2 of Miquel's configuration, which characterizes the Miquelian Laguerre planes, is satisfied. From this perspective, elation Laguerre planes are 'closest' to the Miquelian planes. Finally, finite elation Laguerre planes of odd order $q$ are also equivalent to translation generalized quadrangles of order $q$ with an antiregular point, see [2] and [10]. All of this indicates that elation Laguerre planes form a nice subclass of Laguerre planes and that, if there are finite non-ovoidal Laguerre planes, elation Laguerre planes certainly are a natural class to look for them.

In this paper we conduct a computer search for elation Laguerre planes of order 16, the first order not covered by previous results, and show that each such plane is, in fact, ovoidal. The search results also confirm the numbers of hyperovals through certain points in the dual translation planes of order 16 given in [15]. Section 2 gives a summary of facts about Laguerre planes and in particular elation Laguerre planes of order 16. In section 3 we briefly review the classification of (dual) translation planes of order 16 and their hyperovals and in the last section the method of the computer search and its results are presented.

\section{Some results on elation Laguerre planes}

Explicitly, a finite Laguerre plane $\mathcal{L}=(P, \mathcal{C}, \mathcal{G})$ of order $n$ consists of a set $P$ of $n(n+1)$ points, a set $\mathcal{C}$ of $n^{3}$ circles and a set $\mathcal{G}$ of $n+1$ generators (where circles and generators are both subsets of $P$ ) such that the following three axioms are satisfied:

(G) $\mathcal{G}$ partitions $P$ and each generator contains $n$ points.

(C) Each circle intersects each generator in precisely one point.

$(\mathrm{J})$ Three points no two of which are on the same generator can be uniquely joined by a circle. 
The internal incidence structure $\mathcal{A}_{p}$ at a point $p$ of a Laguerre plane has the collection of all points not on the generator through $p$ as point set and, as lines, all circles passing through $p$ (without the point $p$ ) and all generators not passing through $p$. From the definition of a Laguerre plane it readily follows that each internal incidence structure is an affine plane, the derived affine plane at $p$. $\mathcal{A}_{P}$ extends to a projective plane $\mathcal{P}_{p}$, which we call the derived projective plane at $p$, for short.

A circle $C$ not incident with the distinguished point $p$ induces an oval in $\mathcal{P}_{p}$ by deleting the unique point incident with $C$ and the generator of $p$ and adding the point $\omega$ at infinity that corresponds to the set of generators of $\mathcal{L}$. Note that all ovals arising in this way from circles of $\mathcal{L}$ pass through the common point $\omega$ and have the line at infinity of $\mathcal{P}_{p}$ (with respect to $\mathcal{A}_{p}$ ) as a tangent. Thus a Laguerre plane corresponds to a projective plane with enough of these ovals, that mutually intersect in at most two affine points. This planar description of a Laguerre plane must then be extended by the points of one generator where one has to adjoin a new point to each line and to each oval of the projective plane as above.

Each oval in a projective plane of even order has a nucleus and adjoining this point extends the oval to a hyperoval. In case of ovals that come from circles of a Laguerre plane of even order, the nucleus is on the line at infinity. As before, a Laguerre plane of even order then corresponds to a projective plane with sufficiently many hyperovals through a common point such that any two different ones of these hyperovals intersect in at most four points. This is the basic link between Laguerre planes of even order and projective planes and their hyperovals exploited for our search.

Using the celebrated result of Segre [16] that every oval in a finite Desarguesian projective plane of odd order is a conic, it was shown in [3] or [14], VII.2, that a finite Laguerre plane of odd order that admits a Desarguesian derivation is Miquelian. For small orders this and the results of [19] and [20] imply the following.

THEOREM 1 A Laguerre plane of order at most ten is ovoidal and, in fact, Miquelian except in case of order eight.

An automorphism of a Laguerre plane $\mathcal{L}$ is a permutation of the point set that maps circles onto circles and generators to generators. All automorphisms of $\mathcal{L}$ form a group $\Gamma$ with respect to composition, the automorphism group $\Gamma=\Gamma(\mathcal{L})$ of $\mathcal{L}$. The collection of all automorphisms that fix each generator globally but fix no circle, together with the identity forms a normal subgroup $\Delta_{e}$, see [18]. In case of an elation Laguerre plane $\Delta_{e}$ has maximal order and is the elation group of $\mathcal{L}$ so that $\Delta_{e}$ acts simply transitively on the set of circles. An element of $\Delta_{e}$ induces an elation with center $\omega$ in each derived projective plane at one of its fixed points. Indeed, one has the following, compare [17].

PROPOSITION 2 Each derived projective plane of a finite elation Laguerre plane is a dual translation plane with translation center the point $\omega$ at infinity of vertical lines and has prime power order. An elation Laguerre plane of prime order is Miquelian.

Each oval induced by a circle of an elation Laguerre plane in a derived projective plane passes through the translation center $\omega$ and has the line at infinity as a tangent. 
Note that the first order of a finite elation Laguerre plane not covered by Theorem 1 and Proposition 2 is order 16.

Extending the usual representation of dual translation planes, a description of elation Laguerre planes in terms of two matrix-valued maps was developed in [18], Theorem 3, see also [21]. In particular, for order 16 we have the following representation of elation Laguerre planes.

THEOREM 3 Let $\mathcal{L}=(P, \mathcal{C}, \mathcal{G})$ be a elation Laguerre plane of order 16 . There is a divisor $m$ of 4 , a field $\mathbb{F}=G F\left(2^{4 / m}\right)$ of order $2^{4 / m}$, a symbol $\infty \notin \mathbb{F}^{m}$, and a matrixvalued map $D: \mathbb{F}^{m} \cup\{\infty\} \rightarrow M(3 m, m ; \mathbb{F})$ where $M(3 m, m ; \mathbb{F})$ denotes the set of all $3 m \times m$ matrices over $\mathbb{F}$ such that $\mathcal{L}$ can be represented in the following form. The point set is

$$
P=\left(\mathbb{F}^{m} \cup\{\infty\}\right) \times \mathbb{F}^{m},
$$

the generators are the verticals $\{a\} \times \mathbb{F}^{m}$ of $P$ for $a \in \mathbb{F}^{m} \cup\{\infty\}$, and the set of circles is $\mathcal{C}=\left\{K_{c} \mid c \in \mathbb{F}^{3 m}\right\}$ where a circle $K_{c}$ is described as

$$
K_{c}=\left\{(x, c \cdot D(x)) \in P \mid x \in \mathbb{F}^{m} \cup\{\infty\}\right\} .
$$

The elation group $\Delta_{e}$ consists of all maps

$$
(x, y) \mapsto(x, y+c \cdot D(x)),
$$

for $c \in \mathbb{F}^{3 m}$; also $(x, y) \mapsto(x, r \cdot y)$ for $r \in \mathbb{F}^{*}=\mathbb{F} \backslash\{0\}$ is an automorphism that fixes each generator globally.

More precisely, $D(\infty)=(I O O)^{t}$ where ${ }^{t}$ denotes the transposed matrix and $I$ and $O$ denote the $m \times m$ identity matrix and the $m \times m$ zero matrix respectively. The matrix $D(x), x \in \mathbb{F}^{m}$, can be written as $(A(x) B(x) I)^{t}$ with suitable $m \times m$ matrices $A(x)$ and $B(x)$, where $B$ describes a matrix spread (or spread set) of a translation plane of order 16 (corresponding to the dualization of the derived plane at the infinite point $(\infty, 0)$ ) and $A$ describes a pencil of ovals in the derived projective plane $\mathcal{P}_{(\infty, 0)}$ by

$$
\left\{(x, c \cdot A(x)) \mid x \in \mathbb{F}^{m}\right\} \cup\{\omega\}
$$

for all $c \in \mathbb{F}^{m}, c \neq 0$.

One can further assume that $A(0)=0$ and that each of the ovals above has the $x$-axis $y=0$ (stemming from the circle $K_{0}$ ) as a tangent at $(0,0)$ so that we obtain a pencil of hyperovals $H_{c}=\left\{(x, c \cdot A(x)) \mid x \in \mathbb{F}^{m}\right\} \cup\{\omega,(0)\}$ for $c \in \mathbb{F}^{m}, c \neq 0$, where (0) denotes the point at infinity of the $x$-axis. The restrictions of the hyperovals $H_{c}$ to the set $R=\left(\mathbb{F}^{m} \backslash\{0\}\right) \times\left(\mathbb{F}^{m} \backslash\{0\}\right)$ of affine points neither on the $x$ - nor $y$-axis then form a partition of $R$.

$m=1$ yields an ovoidal Laguerre plane. In this case $\mathbb{F}=G F(16)$ and $A(x)$ is a function $\mathbb{F} \rightarrow \mathbb{F}$ such that $\mathcal{O}_{A}=\{(x, A(x)) \mid x \in \mathbb{F}\} \cup\{\omega\}$ is an oval in the Desarguesian projective plane of order 16 . Then the Laguerre plane is isomorphic to the ovoidal plane 
over $\mathcal{O}_{A}$. Also note that every elation Laguerre plane of order 16 has a representation as in Theorem 3 over $G F(2)$, that is, for $m=4$.

Geometrically, a finite elation Laguerre plane of order 16 as described above is equivalent to a 17 -set of $(m-1)$-dimensional subspaces in the $(3 m-1)$-dimensional projective space over $\mathbb{F}$, see [18], Theorem 4, and also [10] and [2] for related translation generalized quadrangles.

\section{The (dual) translation planes of order 16 and their hyperovals}

The translation planes of order 16 were classified by Dempwolff and Reifart [5]. There are eight distinct translation planes of order 16 :

- the Desarguesian plane PG(2,16),

- the semifield plane SEM4 with kernel $G F(4)$,

- the semifield plane SEM2 with kernel $G F(2)$,

- the Hall plane HALL,

- the Lorimer-Rahilly plane LMRH,

- the Johnson-Walker plane JOWK,

- the derived semifield plane DSFP, and

- the Dempwolff plane DEMP.

The first three of these planes are self-dual and the remaining five planes have duals HALL $^{*}$, LMRH$^{*}$, JOWK$^{*}$, DSFP $^{*}$ and DEMP* ${ }^{*}$ These eight planes are possible candidates for derived planes of an elation Laguerre plane of order 16 .

An explicit description of the seven non-Desarguesian translation planes of order 16 can be obtained from the arithmetic tables for addition and multiplicative loops as given in [4]. (Row 5 in table 5 for the multiplicative loop of LMRH is incorrect.) Lines in these planes are given by equations of the form $y=x m+t$ for $m, t \in S=\{0,1, \ldots, 15\}$ ( $S$ represents the elements of $G F(16)$ as in [4]) where the product $x m$ is computed with respect to the given multiplicative loop and addition + is the same for all eight translation planes as given in Table 1. Using the same addition and multiplication lines in the dual translation planes are, correspondingly, described by $y=m x+t$ for $m, t \in S$.

Cherowitzo [4] classified all hyperovals in the seven non-Desarguesian translation planes of order 16, the Desarguesian case was already established by Hall [8], see also [13]. Since we are in characteristic 2, hyperovals cannot be dualized. However, because the Desarguesian plane and the two semifield planes are self-dual, Cherowitzo's classification also yields all hyperovals in the dual translation planes SEM4 and SEM2. The hyperovals in the remaining five dual translation planes were determined in [15]. 


$\begin{array}{cccccccccccccccc}0 & 1 & 2 & 3 & 4 & 5 & 6 & 7 & 8 & 9 & 10 & 11 & 12 & 13 & 14 & 15 \\ 1 & 0 & 5 & 9 & 15 & 2 & 11 & 14 & 10 & 3 & 8 & 6 & 13 & 12 & 7 & 4 \\ 2 & 5 & 0 & 6 & 10 & 1 & 3 & 12 & 15 & 11 & 4 & 9 & 7 & 14 & 13 & 8 \\ 3 & 9 & 6 & 0 & 7 & 11 & 2 & 4 & 13 & 1 & 12 & 5 & 10 & 8 & 15 & 14 \\ 4 & 15 & 10 & 7 & 0 & 8 & 12 & 3 & 5 & 14 & 2 & 13 & 6 & 11 & 9 & 1 \\ 5 & 2 & 1 & 11 & 8 & 0 & 9 & 13 & 4 & 6 & 15 & 3 & 14 & 7 & 12 & 10 \\ 6 & 11 & 3 & 2 & 12 & 9 & 0 & 10 & 14 & 5 & 7 & 1 & 4 & 15 & 8 & 13 \\ 7 & 14 & 12 & 4 & 3 & 13 & 10 & 0 & 11 & 15 & 6 & 8 & 2 & 5 & 1 & 9 \\ 8 & 10 & 15 & 13 & 5 & 4 & 14 & 11 & 0 & 12 & 1 & 7 & 9 & 3 & 6 & 2 \\ 9 & 3 & 11 & 1 & 14 & 6 & 5 & 15 & 12 & 0 & 13 & 2 & 8 & 10 & 4 & 7 \\ 10 & 8 & 4 & 12 & 2 & 15 & 7 & 6 & 1 & 13 & 0 & 14 & 3 & 9 & 11 & 5 \\ 11 & 6 & 9 & 5 & 13 & 3 & 1 & 8 & 7 & 2 & 14 & 0 & 15 & 4 & 10 & 12 \\ 12 & 13 & 7 & 10 & 6 & 14 & 4 & 2 & 9 & 8 & 3 & 15 & 0 & 1 & 5 & 11 \\ 13 & 12 & 14 & 8 & 11 & 7 & 15 & 5 & 3 & 10 & 9 & 4 & 1 & 0 & 2 & 6 \\ 14 & 7 & 13 & 15 & 9 & 12 & 8 & 1 & 6 & 4 & 11 & 10 & 5 & 2 & 0 & 3 \\ 15 & 4 & 8 & 14 & 1 & 10 & 13 & 9 & 2 & 7 & 5 & 12 & 11 & 6 & 3 & 0\end{array}$

Table 1: Addition table for all eight quasigroups

Recall however, that of all those hyperovals we are only interested in hyperovals that pass through the translation center $\omega$, the point at infinity of the vertical lines (representing generators of the Laguerre plane). Furthermore, using the transitivity of the elation group on the set of circles, we can restrict ourselves to hyperovals that contain the point (0) on the line at infinity and the affine point $(0,0)$.

Table AI in [15] shows that none of the six dual translation planes SEM4, HALL*, LMRH* $^{*}$, JOWK$^{*}, \mathrm{DSFP}^{*}$ and DEMP* has hyperovals through $\omega,(0)$ and $(0,0)$. (The collineation groups of these dual translation planes fix the translation center $\omega$; this occurs as a " 1 " in the orbit numbers and the orbit intersection number 0 below this " 1 " indicates no hyperovals though $\omega$.) For SEM2 there are 18 hyperovals through $\omega,(0)$ and $(0,0)$, see [4], Table 9, and Table 3 in the following section for an explicit list. All these results were confirmed by an independent computer search, see the following section. This, of course, means that only PG(2,16) and SEM2 can possibly occur as derived planes of an elation Laguerre plane of order 16.

PROPOSITION 4 Among the dual translation planes of order 16 only the Desarguesian plane PG(2,16) and the semifield plane SEM2 admit hyperovals through the points $\omega$, (0) and $(0,0)$. Consequently, each derived plane of an elation Laguerre plane of order 16 is Desarguesian or isomorphic to SEM2.

There are two equivalence classes of hyperovals in the Desarguesian plane, cf. [8] and [13]. The first class consists of the regular hyperovals arising from conics plus their nuclei and there are 18 of this kind passing through $\omega,(0)$ and $(0,0)$ and the affine point $(1,1)$. The other class, first discovered by Lunelli and Sce [12], contains 2040 hyperovals through $\omega,(0),(0,0)$ and $(1,1)$. The stabilizer of the so-called Lunelli-Sce hyperovals in PG $(2,16)$ has order 144 . 


\section{The method and results}

For each of the eight dual translation planes an independent computer search for hyperovals containing the points $\omega,(0)$ and $(0,0)$ was carried out. The search procedure used was a straightforward backtracking algorithm. Starting with the three points $(0,0),(0)$ and $\omega$ the general algorithm is to systematically pick an affine point on the vertical line $L_{k}=\{(k, y) \mid y \in S\} \cup\{\omega\}$ (those affine lines passing through $\omega$ ), add it to the list of previously selected points, determine all the points on each of the secant lines which are the joins of the selected points and remove these from the next vertical line $L_{k+1}$. A check is made to see if there are still affine points available on $L_{k+1}$, in which case one is selected and the process is repeated. If all points have already been removed, the last point added to the list is removed and another affine point on the same vertical line $L_{k}$ is selected or moving back further to $L_{k-1}$ etc.

If an affine point can be selected from each vertical affine line in this manner, this set together with the two points at infinity forms a hyperoval. It should be noted that this search is only for hyperovals containing $\omega$ (up to collineations of the projective plane) and thus more restrictive in scope and much shorter than the more general searches in [4] and [15]. Each of the eight translation plane thus could be searched in less than two minutes on a $1.3 \mathrm{GHz} \mathrm{PC}$.

$\begin{array}{ccccccccccccccc}1 & 2 & 3 & 4 & 5 & 6 & 7 & 8 & 9 & 10 & 11 & 12 & 13 & 14 & 15 \\ 2 & 5 & 12 & 3 & 1 & 14 & 10 & 9 & 7 & 11 & 13 & 15 & 8 & 4 & 6 \\ 3 & 10 & 5 & 1 & 12 & 15 & 2 & 13 & 11 & 8 & 14 & 4 & 7 & 6 & 9 \\ 4 & 14 & 7 & 5 & 9 & 1 & 13 & 6 & 3 & 12 & 15 & 2 & 10 & 11 & 8 \\ 5 & 1 & 10 & 7 & 2 & 8 & 6 & 12 & 15 & 14 & 4 & 11 & 3 & 9 & 13 \\ 6 & 15 & 14 & 9 & 13 & 3 & 4 & 10 & 8 & 7 & 2 & 1 & 11 & 12 & 5 \\ 7 & 11 & 13 & 2 & 8 & 4 & 14 & 15 & 5 & 9 & 3 & 10 & 6 & 1 & 12 \\ 8 & 7 & 6 & 13 & 11 & 10 & 15 & 4 & 14 & 5 & 1 & 9 & 12 & 2 & 3 \\ 9 & 4 & 11 & 15 & 14 & 13 & 12 & 3 & 2 & 1 & 10 & 6 & 5 & 8 & 7 \\ 10 & 12 & 2 & 11 & 3 & 7 & 9 & 5 & 4 & 15 & 6 & 8 & 1 & 13 & 14 \\ 11 & 8 & 15 & 14 & 7 & 2 & 3 & 1 & 12 & 6 & 9 & 13 & 4 & 5 & 10 \\ 12 & 3 & 1 & 6 & 10 & 9 & 11 & 7 & 13 & 2 & 8 & 5 & 14 & 15 & 4 \\ 13 & 6 & 9 & 12 & 15 & 5 & 8 & 11 & 10 & 4 & 7 & 14 & 2 & 3 & 1 \\ 14 & 9 & 8 & 10 & 4 & 12 & 1 & 2 & 6 & 13 & 5 & 3 & 15 & 7 & 11 \\ 15 & 13 & 4 & 8 & 6 & 11 & 5 & 14 & 1 & 3 & 12 & 7 & 9 & 10 & 2\end{array}$

Table 2: Multiplication table for SEM2

No hyperovals through $\omega,(0)$ and $(0,0)$ were found in the dual translation planes except for PG $(2,16)$ and SEM2 in agreement with the results in [4] and [15]. The tables for addition and multiplication for SEM2 are reproduced from [4] in Tables 1 and 2 for the convenience of the reader. As in [4] 18 hyperovals containing $\omega,(0)$ and $(0,0)$ were found in SEM2. The affine points of the 18 hyperovals are listed in Table 3 below. Each row represents a hyperoval and an entry $j$ in column $i$ means that the point $(i, j)$ belongs to the corresponding hyperoval. From Table 3 one sees that there is no hyperoval passing 
through the point $(1,4)$. This contradicts the fact that the hyperovals in the pencil we want to construct must form a partition of the set $R$ of affine points neither on the $x$ - nor $y$-axis.

\begin{tabular}{c|cccccccccccccccc} 
& 0 & 1 & 2 & 3 & 4 & 5 & 6 & 7 & 8 & 9 & 10 & 11 & 12 & 13 & 14 & 15 \\
\hline 1 & 0 & 1 & 13 & 5 & 8 & 12 & 7 & 4 & 9 & 2 & 3 & 14 & 11 & 6 & 15 & 10 \\
2 & 0 & 1 & 13 & 6 & 5 & 12 & 15 & 9 & 14 & 11 & 7 & 4 & 10 & 8 & 3 & 2 \\
3 & 0 & 2 & 12 & 3 & 1 & 7 & 10 & 9 & 14 & 6 & 13 & 4 & 8 & 15 & 11 & 5 \\
4 & 0 & 2 & 12 & 15 & 3 & 7 & 11 & 14 & 4 & 8 & 10 & 9 & 5 & 1 & 13 & 6 \\
5 & 0 & 3 & 11 & 6 & 8 & 5 & 1 & 14 & 4 & 2 & 7 & 9 & 10 & 12 & 15 & 13 \\
6 & 0 & 3 & 11 & 12 & 6 & 5 & 15 & 4 & 9 & 10 & 1 & 14 & 13 & 8 & 7 & 2 \\
7 & 0 & 5 & 7 & 2 & 10 & 13 & 12 & 4 & 9 & 1 & 6 & 14 & 3 & 11 & 8 & 15 \\
8 & 0 & 5 & 7 & 10 & 11 & 13 & 6 & 14 & 4 & 15 & 8 & 9 & 1 & 2 & 12 & 3 \\
9 & 0 & 6 & 3 & 10 & 13 & 2 & 12 & 9 & 14 & 7 & 8 & 4 & 1 & 11 & 5 & 15 \\
10 & 0 & 6 & 3 & 11 & 10 & 2 & 5 & 14 & 4 & 1 & 12 & 9 & 15 & 13 & 8 & 7 \\
11 & 0 & 8 & 1 & 2 & 11 & 10 & 5 & 9 & 14 & 15 & 6 & 4 & 3 & 13 & 12 & 7 \\
12 & 0 & 8 & 1 & 11 & 13 & 10 & 6 & 4 & 9 & 7 & 12 & 14 & 15 & 2 & 5 & 3 \\
13 & 0 & 10 & 5 & 3 & 7 & 15 & 11 & 4 & 9 & 12 & 13 & 14 & 8 & 1 & 2 & 6 \\
14 & 0 & 10 & 5 & 7 & 1 & 15 & 13 & 14 & 4 & 6 & 2 & 9 & 12 & 3 & 11 & 8 \\
15 & 0 & 11 & 6 & 7 & 3 & 1 & 10 & 4 & 9 & 8 & 2 & 14 & 12 & 15 & 13 & 5 \\
16 & 0 & 11 & 6 & 15 & 7 & 1 & 13 & 9 & 14 & 12 & 10 & 4 & 5 & 3 & 2 & 8 \\
17 & 0 & 15 & 2 & 5 & 6 & 8 & 1 & 9 & 14 & 10 & 3 & 4 & 11 & 12 & 7 & 13 \\
18 & 0 & 15 & 2 & 12 & 5 & 8 & 7 & 14 & 4 & 11 & 1 & 9 & 13 & 6 & 3 & 10
\end{tabular}

Table 3: Affine parts of hyperovals in SEM2

As an aside note the interesting property that all the 18 hyperovals pass through the set $\{7,8,11\} \times\{4,9,14\}$. More precisely, six of the hyperovals contain the three points $(7,4),(8,9),(11,14)$, another six pass through $(7,9),(8,14)$ and $(11,4)$ and the last six hyperovals contain the three points $(7,14),(8,4),(11,9)$. Also note that $\{0,7,8,11\}$ and $\{0,4,9,14\}$ are additive subgroups.

Hence SEM2 is not possible as a derived plane of an elation Laguerre plane of order 16.

PROPOSITION 5 No pencil of hyperovals can be formed from the 18 hyperovals through $\omega,(0)$ and $(0,0)$ in SEM2. Hence each derived plane of an elation Laguerre plane of order 16 is Desarguesian.

This leaves us with the Desarguesian plane. The above search procedure was modified to yield only those hyperovals containing $\omega,(0),(0,0)$ and also the affine point $(1,1)$. In agreement with well known results on such hyperovals $2058=18+2040$ hyperovals were found. Application of the homologies $\rho_{r}:(x, y) \mapsto(x, r y)$ for $r \in G F(16)^{*}$ then yields all 30870 hyperovals of $\mathrm{PG}(2,16)$ containing $\omega,(0),(0,0)$. Of these 15 hyperovals have to be selected such that their restrictions to the set $R$ of affine points neither on the $x$ - nor $y$-axis form a partition of $R$. 
Clearly, for each hyperoval $H$ the collection $\left\{\rho_{r}(H) \mid r \in G F(16)^{*}\right\}$ is such a pencil that partitions $R$. We call this collection a trivial pencil. But there are non-trivial pencils of hyperovals in $\mathrm{PG}(2,16)$ as well. For example, let

$$
\begin{aligned}
H_{2, r} & =\left\{\left(x, r x^{2}\right) \mid x \in G F(16)\right\} \cup\{\omega,(0)\} \\
H_{8, r} & =\left\{\left(x, r x^{8}\right) \mid x \in G F(16)\right\} \cup\{\omega,(0)\} \\
H_{14, a, r} & =\left\{\left(x, r\left((x+a)^{14}+a^{14}\right)\right) \mid x \in G F(16)\right\} \cup\{\omega,(0)\}
\end{aligned}
$$

for $a, r \in G F(16), r \neq 0$. These are all the regular hyperovals (i.e., conics plus nucleus) containing $\omega,(0),(0,0)$. Then

$$
\left\{H_{2, r} \mid r \in G F(16) \backslash G F(16)^{3}\right\} \cup\left\{H_{8, r} \mid r \in G F(16)^{3}, r \neq 0\right\}
$$

where $G F(16)^{3}=\left\{x^{3} \mid x \in G F(16)\right\}$ denotes the set of all cubes in $G F(16)$ or

$$
\left\{H_{14, a, a} \mid a \in G F(16)\right\}
$$

are non-trivial pencils of hyperovals.

For elation Laguerre planes of order 16, however, we need linear pencils of hyperovals, that is, if $H_{i}=\left\{\left(x, h_{i}(x)\right) \mid x \in G F(16)\right\} \cup\{\omega,(0)\}, i=1,2$, are any two different hyperovals in such a pencil for suitable permutation polynomials $h_{1}$ and $h_{2}$, then $h_{1}+h_{2}$ also represents a hyperoval in the pencil. Note that the two pencils mentioned above are not linear. Indeed, one has the following.

PROPOSITION 6 If a linear pencil in PG(2,16) contains two regular hyperovals $H_{1}$ and $H_{2}$ through the points $\omega,(0)$ and $(0,0)$, then $H_{2}=\rho_{r}\left(H_{1}\right)$ for some $r \in G F(16)^{*}$. In particular, a linear pencil of regular hyperovals in $P G(2,16)$ is trivial.

Proof. We apply a characterization of hyperoval describing polynomials given in [6]. It says that the set $\{(x, f(x)) \mid x \in G F(q)\} \cup\{(0), \omega\}$ where $f$ is a polynomial $f$ of degree at most $q-2$ satisfying $f(0)=0$ and $f(1)=1$ is a hyperoval in the Desarguesian projective plane of even order $q$ if and only if the coefficient of $x^{c}$ in $f(x)^{b}$ modulo $x^{q}-x$ is zero for all pairs of integers $(b, c)$ such that $1 \leq b \leq c \leq q-1, b \neq q-1$ and all terms appearing in the binary expansion of $b$ also appear in the binary expansion of $c$.

Since the coefficient of $x^{3}$ in $\left(r x^{8}+s x^{2}\right)^{3}$ modulo $x^{16}-x$ is $r^{2} s \neq 0$ for $r, s \neq 0, H_{8, r}$ and $H_{2, s}$ cannot occur together in a linear pencil by the characterization above. Likewise, the coefficients of $x^{15}$ and $x^{11}$ in $\left(r\left((x+a)^{14}+a^{14}\right)+s\left((x+b)^{14}+b^{14}\right)\right)^{3}$ modulo $x^{16}-x$ are $r s(r+s)(a+b)^{12}$ and $r s\left(r a^{4}+s b^{4}\right)(a+b)^{12}$, at least one of which is non-zero for $r, s \neq 0$, $a \neq b$. Hence, $H_{14, a, r}$ and $H_{14, b, s}$ cannot occur together in a linear pencil for $a \neq b$. Finally, the coefficients of $x^{15}$ in $\left(r\left((x+a)^{14}+a^{14}\right)+s x^{2}\right)^{3}$ and $\left(r\left((x+a)^{14}+a^{14}\right)+s x^{8}\right)^{3}$ modulo $x^{16}-x$ are $r^{2} s \neq 0$ and $r s^{2} \neq 0$ so that $H_{14, a, r}$ and $H_{2, s}$ or $H_{8, s}$ cannot occur together in a linear pencil.

A straightforward computer search looked for different hyperovals $H_{1}, H_{2}$ in the list of 2058 hyperovals found previously such that the set $\left.\rho_{1 /(1+r)}\left(H_{1}+\rho_{r}\left(H_{2}\right)\right)\right)$ is again in 
the list of hyperovals for some $r \in G F(16), r \neq 0,1$. Not surprisingly, given Proposition 6 and the intricate algebraic properties of the Lunelli-Sce hyperovals (compare [9]), none where found. Hence, in order to obtain hyperovals in this way we must have $H_{1}=H_{2}$. But then we obtain a trivial pencil.

PROPOSITION 7 A linear pencil of hyperovals through the points $\omega,(0)$ and $(0,0)$ in $P G(2,16)$ is trivial, that is, of the form $\left\{\rho_{r}(H) \mid r \in G F(16)^{*}\right\}$ for one fixed hyperoval $H$.

Of course, this means that the underlying elation Laguerre plane can be represented over $G F(16)$, that is, $m=1$, and the Laguerre plane is ovoidal. Hence, we obtain our final result.

THEOREM 8 A finite elation Laguerre plane of order 16 is ovoidal.

As a final remark note that a Laguerre plane of order 16 induces $16^{3}-16^{2}=3840$ hyperovals in each of its derived projective planes. Hence, in order that a particular projective plane of order 16 can possibly occur as a derived plane it is necessary that it contains at least 3840 hyperovals all of which must pass through a common point. Looking at the total numbers of hyperovals as given in Table II in [15] (although there seem to be some misprints which lead to inconsistencies with Table I) the above observation immediately excludes nine of the 22 projective planes of order 16 as candidates for derived projective planes of a Laguerre plane of order 16. Taking account of the orbit intersection numbers in Tables AI and AII in [15], some of the other total numbers of hyperovals also seem to be too low to support sufficiently many hyperovals through a common point. Therefore and given the negative results from [7] we conjecture that only PG(2,16) can occur as a derived plane of a Laguerre plane of order 16. Furthermore, because of the difficulties of combining non-trivial pencils of hyperovals to a Laguerre plane (see [20] for the case of order 8), that in this case the Laguerre plane must be ovoidal.

\section{References}

[1] R.C. Bose and K.A. Bush, Orthogonal arrays of strength two and three, Ann. Math. Statistics 23 (1952), 508-524.

[2] L.R.A. Casse, J.A. Thas and P.R. Wild, $\left(q^{n}+1\right)$-sets of PG $(3 n-1, q)$, generalized quadrangles and Laguerre planes, Simon Stevin 59 (1985), 21-42.

[3] Y. Chen and G. Kaerlein, Eine Bemerkung über endliche Laguerre- und Minkowski-Ebenen, Geom. Dedicata 2 (1973), 193-194.

[4] W.E. Cherowitzo, Hyperovals in the translation planes of order 16, J. Combin. Math. and Combin. Comp. 9 (1991), 39-55. 
[5] U. Dempwolff and A. Reifart, The classification of the translation planes of order 16, Geom. Dedicata 15 (1983), 137-153.

[6] D.G. Glynn, A condition for the existence of ovals in $P G(2, q), q$ even, Geom. Dedicata 32 (1989), 247-252.

[7] D.G. Glynn and G.F. Steinke, Translation Laguerre planes of order 16, Europ. J. Comb. 14 (1993), 529-539.

[8] M. Hall, Jr, Ovals in the Desarguesian plane of Order 16, Ann. Mat. Pura Appl. (4) 102 (1975), 159-176.

[9] J.W.P. Hirschfeld, Projective Geometries over Finite Fields, 2nd edition, Clarendon Press, Oxford, England, 1998.

[10] M. Joswig, Pseudo-ovals, elation Laguerre planes, and translation generalized quadrangles, Beitr. Algebra Geom. 40 (1999), 141-152.

[11] N. Knarr, A geometric characterization of elation Laguerre planes, Arch. Math. 78 (2002), 162-165.

[12] L. Lunelli and M. Sce, $k$-archi completi nei piani proietivi desarguesiani di rango 8 e 16 , Centro Calcoli Numerici, Politecnico di Milano, 1958.

[13] C.M. O'Keefe and T. Penttila, Hyperovals in PG(2,16), Europ. J. Combin. 12 (1991), $51-59$.

[14] S.E. Payne and J.A. Thas, Generalized quadrangles with symmetry, Part II, Simon Stevin 49 (1976), 81-103.

[15] T. Penttila, G.F. Royle, and M.K. Simpson, Hyperovals in the known projective planes of order 16, J. Combin. Des. 4 (1996), 59-65.

[16] B. Segre, Ovals in a finite projective plane, Canad. J. Math. 7 (1955), 414-416.

[17] G.F. Steinke, Semiclassical 4-dimensional Laguerre planes, Forum Math. 2 (1990), 233-247.

[18] G.F. Steinke, On the structure of finite elation Laguerre planes, J. Geom. 41 (1991), 162179.

[19] G.F. Steinke, A remark on Benz planes of order 9, Ars Combin. 34 (1992), 257-267.

[20] G.F. Steinke, Finite Laguerre planes of order 8 are ovoidal, J. Combin. Th., Series A, 102 (2003), 143-162.

[21] J. A. Thas, The $m$-dimensional projective space $S_{m}\left(M_{n}(G F(q))\right)$ over the total matrix algebra $M_{n}(G F(q))$ over the $n \times n$ matrices with elements in the Galois field $G F(q)$, Rend. Mat. 4 (1971), 459-532.

Günter F. Steinke, Department of Mathematics and Statistics, University of Canterbury, Private Bag 4800, Christchurch 8020, New Zealand

e-mail: G.Steinke@math.canterbury.ac.nz 